\title{
Application of quantitative techniques for the assessment of gastric atrophy
}

Atrophic gastritis is seen as the result of long standing Helicobacter pylori infection and recent studies have shown that circulating IgG antibodies to CagA are associated with this condition. Gastric atrophy is also seen in patients with pernicious anaemia caused by an immunological derangement associated with parietal cell antibodies. Nonimmunological causes of atrophic gastritis include chronic alcoholism, bile reflux, drugs, partial gastrectomy, chronic pancreatitis, and liver cirrhosis. The clinical importance of gastric atrophy is that it significantly increases the risk for the development of gastric carcinoma. The prevalence of gastric atrophy is very variable but it can be detected in up to $25 \%$ of patients referred for upper gastrointestinal endoscopy. In the Sydney system of classification of gastritis a four grade scale is used: no atrophy and mild, moderate, or severe atrophy. Despite this, agreement among histopathologists for the recognition and grading of gastritis remains poor. The paper by Van Grieken et al in the January issue of this journal describes a new stereological method (point counting technique) for grading gastric atrophy in body type mucosa by an interactive image analysis system (QPRODIT). ${ }^{1}$ Using an updated Sydney system of classification of gastritis ${ }^{2}$ they concluded that the point counting technique is a powerful and reproducible tool for the quantitative analysis of mucosal atrophy irrespective of the $H$ pylori status. It is also a reliable method for measuring the volume percentage of stroma and inflammation in the body mucosa. The volume percentage of glands in the body mucosa reported by Van Grieken et al was approximately $50 \%$ in grade II atrophy and $30 \%$ in grade III atrophy as assessed by the Sydney system. Similar results were reported previously, ${ }^{3}$ using different quantitative techniques based upon syntactic structure analysis. A high degree of accuracy is well known in quantitative pathology in comparison with routine morphology, but unlike quantitative techniques, subjective assessment of gastric atrophy of the antral mucosa produced variable results with poor observer agreement even in the hands of specialists in gastrointestinal pathology. ${ }^{4}$ This might be caused in part by lack of agreement among histopathologists on defining criteria for gastric atrophy.

Currently, gastric atrophy is defined by loss of glands in the gastric mucosa. The updated Sydney system has adopted this definition and in addition uses schematic diagrams to help histopathologists in recognition and grading. If this definition is accepted, then loss of glands implies loss of the entire glandular structure, including the loss of proliferative zone and stem cells. Loss of stem cells is a permanent event and one would not expect regeneration or intestinal metaplasia, dysplasia, or carcinoma to develop in such patients. However, epidemiological studies have shown a strong correlation between gastric atrophy, intestinal metaplasia, and the development of carcinoma. In addition, recent clinical trials ${ }^{5}$ have shown reversal of fundic gastric atrophy after eradication of $\mathrm{H}$ pylori, and histological studies and clinical experience have shown a correlation between proliferation, intestinal metaplasia, and gastric atrophy. Intestinal metaplasia is not seen in normal stomach so that its presence indicates partial or complete loss of the normal epithelium that occurs in many forms of gastritis including gastric atrophy. Van Grieken et al found that intestinal metaplasia was present in $48 \%$ of patients with pronounced atrophy and in only one patient with moderate atrophy, confirming the hypothesis that intestinal metaplasia is a more advanced stage than gastric atrophy in the histological sequence leading to gastric cancer. ${ }^{1}$ In a recent paper, ${ }^{3}$ we have shown that gastric atrophy is characterised by architectural changes manifested by variation in the volume and irregularity in the shape, branching, and spacing of the glands. These changes would not occur as the result of loss of glands alone, but other factors in the lamina propria, including myofibroblasts and inflammatory cells, might react with the gland to produce such structural alterations. The paper reported by Van Grieken et al is therefore important not only for assessing the volume percentages of glands, but also for assessing the volume percentage of other components of the lamina propria, including myofibroblasts. In our opinion, gastric atrophy is characterised by changes in both the epithelium and stroma of body-type and antral-type gastric mucosa, with partial or complete loss of the glandular epithelium, which may lead to architectural, metaplastic, proliferative, and functional changes. Both partial and complete loss of the glandular epithelium may be seen in the same patient in different parts of the stomach depending upon the nature and duration of the insult to the gastric mucosa.

The sites of biopsy are important for the accurate diagnosis of gastric atrophy. In the updated Sydney system, ${ }^{2}$ five biopsy sites were recommended including the incisura. However, others ${ }^{6}$ recommend that four biopsies should be taken from the mid antrum and mid body of the lesser curvature and the mid antrum and mid body of the greater curvature. Classifying gastric atrophy into two grades-low grade and high grade - has been reported using quantitative $^{3}$ and non-quantitative techniques. ${ }^{4}$ The interobserver agreement among pathologists was better when two grades were used rather than three. It is generally believed that high grade gastric atrophy is associated with intestinal metaplasia and dysplasia and has prognostic clinical relevance in comparison with low grade gastric atrophy, which might not be clinically important.

In conclusion, the assessment of gastric atrophy requires proper biopsy sampling, recognition of changes in both the glandular epithelium and the lamina propria, and an accurate technique for grading the atrophy. In addition, for a grading system to be of most value, there should be a good clinicopathological correlation. Although the development of quantitative techniques has aided and supported subjective routine diagnosis, their role in the assessment of prognosis and planning for treatment should be evaluated.

A M ZAITOUN

Department of Histopathology, University Hospital, Queen's Medical Centre, Nottingham NG7 2UH, UK

abd.Zaitoun@mail.qmouh-tr.trent nhs.uk 
C O RECORD

Department of Medicine, The Royal Victoria Infirmary, Newcastle upon Tyne NE1 4LP, UK

1 Van Grieken NCT, Weiss MM, Meijer GA, et al. Rapid quantitative assessment of gastric corpus atrophy in tissue sections. 7 Clin Pathol 2001;54:63ment

2 Dixon MF, Genta RM, Yardley JH, et al. Classification and grading of gastritis; the updated Sydney system. Am $\mathcal{F}$ Surg Pathol 1996;20:1161-81.
3 Zaitoun AM, Mardini H Al, Record CO. Quantitative assessment of gastric atrophy using the syntactic structure analysis. 7 Clin Pathol 1998;51:895atroph

4 Offerhaus GJA, Price AB, Haot J, et al. Observer agreement on the grading of gastric atrophy. Histopathology 1999;34:320-5.

5 Tucci A, Poli 1, Tosetti C, et al. Reversal of fundic atrophy after eradication of Helicobcater pylori. Am f Gastroenterol 1998;93:1425-31.

6 Satoh K, Kimura K, Taniguchi Y, et al. Biopsy sites suitable for the diagnosis of Helicobacter pylori infection and the assessment of the extent of atrophic gastritis. Am f Gastroenterol 1998;93:569-73.

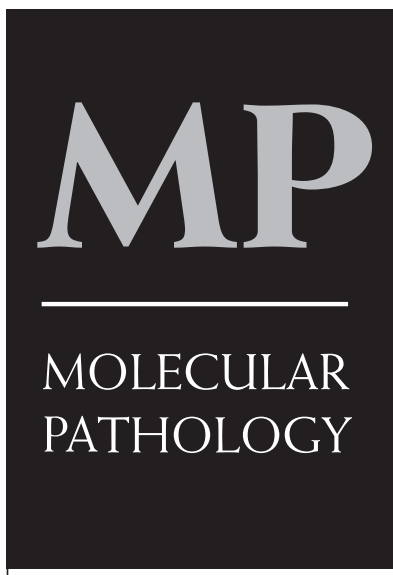

Contents
February 2001 Vol 54 No 1

\section{Reviews}

1 Demystified... Adhesion molecule deficiencies D Inwald, E G Davies, N Klein

8 Microarrays under the microscope $S E$ Wildsmith, $F$ F Elcock

\section{Papers}

17 Correlation between apoptosis macroarray gene expression profiling and histopathological lymph node lesions $\mathcal{J}$ P Dales, $\mathcal{F}$ Plumas, F Palmerini, E Devilard, T Defrance, A Lajmanovich, V Pradel, F Birg, L Xerri

24 Rapid real time PCR to distinguish between high risk human papillomavirus types 16 and $18 H$ H Cubie, A L Seagar, E McGoogan, $\mathcal{F}$ Whitehead, A Brass, $M \mathcal{F}$ Arends, $M W$ Whitley

30 Insulin-like growth factors I and II induce cell death in Wilms's tumour cells $M$ Granérus, $A$ fohannisson, P Ekblom, W Engström

\section{Editorial}

36 The University of Halle through the centuries W Zumkeller

\section{Abstracts}

38 Abstracts

Miscellanea

55 Book Reviews 